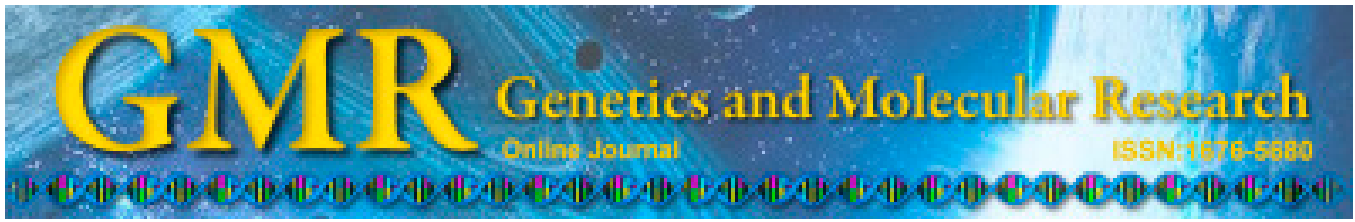

\title{
Prevalence and genotype distribution of human papillomavirus in women in the Henan Province
}

\author{
Y.-F. Zhi' ${ }^{1}$, X.-X. Cha ${ }^{2}$, X.-F. Li' ${ }^{1}$ C. Qiu ${ }^{1}$ and S.-H. Rong ${ }^{1}$ \\ ${ }^{1}$ Department of Cytopathology, \\ Third Affiliated Hospital of Zhengzhou University, Zhengzhou, China \\ ${ }^{2}$ Department of Pharmacology, Basic Medical College of Zhengzhou University, \\ Zhengzhou, China \\ Corresponding author: Y.-F. Zhi \\ E-mail: zhiyanfang_zyf@163.com
}

Genet. Mol. Res. 14 (2): 5452-5461 (2015)

Received October 24, 2014

Accepted January 19, 2015

Published May 22, 2015

DOI http://dx.doi.org/10.4238/2015.May.22.15

ABSTRACT. We studied human papillomavirus (HPV) prevalence and genotype distribution among women in the Henan Province to provide epidemiological data as a means of preventing cervical cancer and developing a vaccine. A total of 14,873 samples were genotyped by using polymerase chain reaction reverse dot-blot. The overall HPVpositive rate in the sample was $23.98 \%$ (3566/14873), of which $69.01 \%$ (2461/3566) were infected with high-risk HPV types and $17.33 \%$ (618/3566) with low-risk types. Eighteen high-risk HPV types were detected; HPV $16(16.73 \%)$ was the most common, followed by 58 $(10.17 \%), 52$ (9.11\%), $56(6.48 \%), 66(5.76 \%), 33(4.74 \%), 68$ (3.92\%), $31(3.60 \%), 53(3.13 \%), 59(3.00 \%), 35(2.53 \%), 51(2.00 \%)$, $73(1.08 \%), 45(0.94 \%), 83(0.84 \%), 39(0.69 \%), 18(0.61 \%)$, and MM4 (0.04\%). Four low-risk HPV types were detected; HPV 43 (11.34\%) was the most common, followed by $6(5.17 \%), 42$ (4.76\%), and $11(3.35 \%)$. Type 44 was not detected. Among the women positive for HPV, $71.17 \%$ (2538/3566) had a single type of infection; of these, $54.66 \%(1949 / 3566)$ had high-risk and $16.52 \%$ (589/3566) had lowrisk infections. A total of $28.83 \%$ (1028/3566) had multiple HPV infec- 
tions, of which 20.11\% (717/3566) had double HPV infections. One peak in HPV prevalence occurred among women younger than age 25; a second peak occurred among women older than age 55. The overall prevalence of HPV infection in the Henan Province was 23.98\%, of which the most common type was high-risk HPV and a single type of infection. The leading genotypes were HPV 16, 43, 58, 52, and 56.

Key words: Human papillomavirus; Genotypes; Vaccine; Cervical cancer

\section{INTRODUCTION}

Epidemiology and basic research have confirmed that human papillomavirus (HPV) infection is a major cause of cervical intraepithelial neoplasia and cervical cancer (Pierce Campbell et al., 2012; Ward et al., 2012). HPV infection has a strong regionalism, and HPV infection rates and distribution in different countries or regions are different (Clifford et al., 2005); therefore, studying the distribution of HPV in a particular region provides important guidance for developing and applying vaccines. To understand the infection status and distribution characteristics of HPV in women in the Henan Province, we undertook HPV genotyping in 14,873 women in the Province to provide a theoretical basis for prevention and treatment of cervical cancer and development of HPV vaccines.

\section{MATERIAL AND METHODS}

\section{Research subjects}

The database consisted of 14,873 women from the outpatient services of the departments of gynecology, reproductive medicine, women's health, and the health physical of The Third Affiliated Hospital of Zhengzhou University between February 2012 and May 2013. Inclusion criteria were women who had lived in the Henan Province for more than 5 years and were sexually active. Participants' ages mainly ranged from 25 to 45 years, with a median age of 36 years; the youngest was 14 and the oldest was 88 .

\section{Main reagents and equipment}

An HPV genotyping detection kit [polymerase chain reaction (PCR)/reverse dot blot] reading instrument was used (Yaneng Bio Technology Co., Ltd.). It can detect 18 high-risk subtypes: HPV 16, 18, 31, 33, 35, 45, 51, 52, 53, 56, 58, 59, 66, 68, 73, 83, and MM4, and 5 kinds of low-risk subtypes: HPV 6, 11, 42, 43, and 44.

\section{Experimental method}

\section{Draw materials}

We placed the specific sampling brush for HPV into the patient's cervix, uniaxially rotated the brush 4 to 5 times, put the brush head into the elution tube, broke the brush handle off, tightened the lid, and made marks. 


\section{DNA extraction}

We ensured the brush was eluted sufficiently and then wiped it, transferring $1 \mathrm{~mL}$ of eluent into a $1.5-\mathrm{mL}$ centrifuge tube. The tube was centrifuged for $10 \mathrm{~min}$ at 13,000 revolutions per min (rpm); we then discarded the supernate and retained the precipitated cells in the bottom of the tube. We then added $50 \mu \mathrm{L}$ of lysate, suspended the sedimentation, placed it in a boiling water bath for $30 \mathrm{~min}$, centrifuged it for $10 \mathrm{~min}$ at 13,000 rpm, and retained the supernate for later use.

\section{PCR amplification}

We took the PCR reaction tube, centrifuged it for $2 \mathrm{~s}$ at $5000 \mathrm{rpm}$, and added $5 \mu \mathrm{L}$ extracted DNA. A reaction system of $25 \mu \mathrm{L}$ was amplified under the following conditions: $50^{\circ} \mathrm{C}$ for $15 \mathrm{~min} ; 95^{\circ} \mathrm{C}$ for $10 \mathrm{~min} ; 94^{\circ} \mathrm{C}$ for $30 \mathrm{~s} ; 42^{\circ} \mathrm{C}$ for $90 \mathrm{~s} ; 72^{\circ} \mathrm{C}$ for $30 \mathrm{~s} ; 40$ cycles; $72^{\circ} \mathrm{C}$ for $5 \mathrm{~min}$.

\section{Hybridization}

We took a $15-\mathrm{mL}$ plastic centrifuge tube, added 5-6 $\mathrm{mL}$ of liquid $\mathrm{A}$ and $25 \mu \mathrm{L}$ of the PCR product, placed it in a boiling water bath for $10 \mathrm{~min}$, and hybridized it for at least $1.5 \mathrm{~h}$ in a hybrid box at $51^{\circ} \mathrm{C}$.

\section{Membrane wash}

We removed the membrane strip to a $50-\mathrm{mL}$ tube containing preheated solution $\mathrm{B}$, which was shaken gently and washed for $5 \mathrm{~min}$ at $51^{\circ} \mathrm{C}$.

\section{Color reaction}

We removed the membrane strip, placed it into the incubation medium containing liquid A and POD (2000:1), shook it gently, and incubated it for $30 \mathrm{~min}$ at room temperature, after which the incubation medium was discarded. The membrane strip was then shaken and washed with liquid A at room temperature twice, each time for $5 \mathrm{~min}$. We washed the membrane with liquid $\mathrm{C}$ for 1-2 min at room temperature while the color liquid was being prepared. The membrane strip was then dipped into a color-substrate solution to dark color for $30 \mathrm{~min}$ in dark. We read the HPV genotyping results with the previously mentioned reading instrument.

\section{Statistical analysis}

Data were analyzed using the SPSS 17.0 statistical software. F and chi-square tests were compared in every group. A P value $<0.05$ was considered to be statistically significant.

\section{RESULTS}

\section{Detection of the genotype of HPV infection}

The overall HPV-positive rate in women was $23.98 \%(3566 / 14,873)$. Of the 23 sub- 
types, 22 were measured; the low-risk HPV 44 type was not measured. From the frequency of detected subtypes, high-risk HPV genotypes accounted for $75.38 \%$ of samples $(3690 / 4895)$, with HPV rankings from high to low as follows: 16 (16.73\%), $58(10.17 \%), 52(9.11 \%), 56$ (6.48\%), 66 (5.76\%), 33 (4.74\%), 68 (3.92\%), 31 (3.60\%), $53(3.13 \%), 59(3.00 \%), 35(2.53 \%)$, $51(2.00 \%), 73(1.08 \%), 45(0.94 \%), 83(0.84 \%), 39(0.69 \%), 18(0.61 \%)$, and MM4 (0.04\%). The low-risk HPV phenotypes accounted for $24.62 \%$ of samples $(1205 / 4895)$, with the HPV rankings from high to low as follows: 43 (11.34\%), $6(5.17 \%), 42(4.76 \%), 11(3.35 \%)$, and $44(0.00 \%)$ (the low-risk HPV 44 genotype was not measured). The comprehensive rankings of high-risk and low-risk were as follows: 16 (16.73\%), 43 (11.34\%), 58 (10.17\%), 52 (9.11\%), 56 (6.48\%), $66(5.76 \%), 6(5.17 \%), 42(4.76 \%), 33(4.74 \%), 68(3.92 \%), 31(3.60 \%)$, $11(3.35 \%), 53(3.13 \%), 59(3.00 \%), 35$ (2.53\%), 51 (2.00\%), 73 (1.08\%), $45(0.94 \%), 83$ $(0.84 \%), 39(0.69 \%), 18(0.61 \%)$, MM $4(0.04 \%)$, and $44(0.00 \%)$. For the distribution of the 14,873 HPV infection cases, see Table 1 and Figure 1.

\begin{tabular}{lccc} 
Table 1. Distribution of HPV infection. & & \\
\hline Groups & HPV subtype & Cases (N) & Proportion (\%) \\
\hline High-risk & 16 & 819 & 16.73 \\
& 18 & 30 & 0.61 \\
& 31 & 176 & 3.60 \\
& 33 & 232 & 4.74 \\
& 35 & 124 & 0.53 \\
& 39 & 34 & 0.94 \\
& 45 & 46 & 2.00 \\
& 51 & 98 & 9.11 \\
& 52 & 446 & 3.13 \\
& 53 & 153 & 6.48 \\
& 56 & 317 & 10.17 \\
& 58 & 498 & 3.00 \\
& 59 & 147 & 5.76 \\
& 66 & 282 & 3.92 \\
& 68 & 192 & 1.08 \\
& 73 & 53 & 0.84 \\
& 83 & 41 & 0.04 \\
& MM4 & 2 & 75.38 \\
& Total & 369 & 5.17 \\
& 6 & 253 & 3.35 \\
& 11 & 164 & 4.76 \\
& 42 & 233 & 11.34 \\
& 43 & 555 & 0.00 \\
& 44 & 0 & 24.62 \\
\hline
\end{tabular}

HPV, human papilloma virus in mixed infections, each subtype was individually counted. A total of 4895 subtypes were determined.

\section{Single and multiple HPV infections}

In 3566 cases of infected women, high-risk infection accounted for $69.01 \%$ of infections (2461/3566), low-risk infections accounted for 17.33\% (618/3566), and mixed HPV infection accounted for $13.66 \%(487 / 3566)$.

In 3566 cases of infected women, more than $1 \mathrm{HPV}$ infection (2538 cases) accounted for $71.17 \%$ of infections (2538/3566), in which high-risk infection accounted for $54.66 \%$ (1949/3566) and low-risk infection accounted for $16.52 \%$ (589/3566). A total of 1028 cases of multiple HPV infections accounted for $28.83 \%$ (1028/3566), in which double infection ac- 
counted for $20.11 \%$ (717/3566). Of the high-risk double infection cases, the numbers significantly decreased as the number of genotypes increased. Eight particular HPV subtypes were simultaneously detected in multiple infections the most often (Table 2).

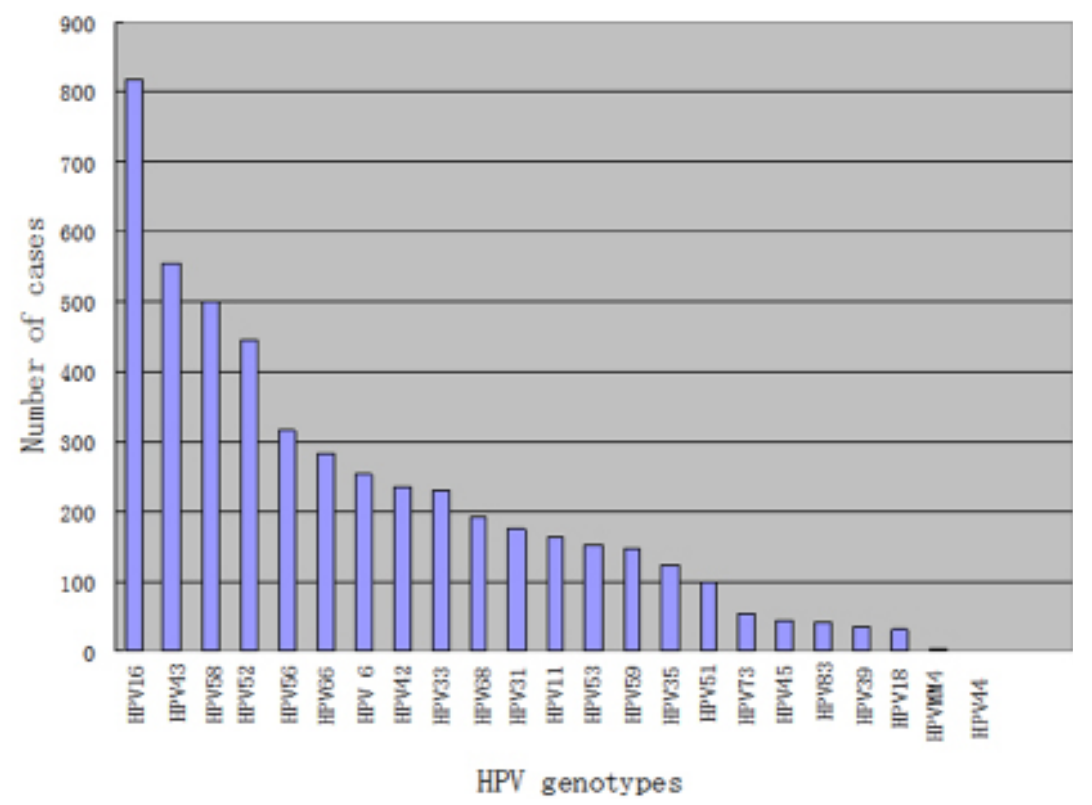

Figure 1. Distribution of human papilloma virus genotypes in the Henan province.

Table 2. Distribution of types of human papilloma virus infection [N (\%)].

\begin{tabular}{lccrr}
\hline Type of infection & No. of cases & Low-risk & High-risk & Mixed \\
\hline Single & $2538(71.17 \%)$ & $589(16.52 \%)$ & $1949(54.66 \%)$ & - \\
Double & $717(20.11 \%)$ & $26(0.73 \%)$ & $414(11.61 \%)$ & $277(7.77 \%)$ \\
Triple & $198(5.55 \%)$ & $3(0.08 \%)$ & $72(2.02 \%)$ & $123(3.45 \%)$ \\
Quadruple & $63(1.77 \%)$ & $0(0.00 \%)$ & $21(0.59 \%)$ & $42(1.18 \%)$ \\
Quintuple & $34(0.95 \%)$ & $0(0.00 \%)$ & $5(0.14 \%)$ & $29(0.81 \%)$ \\
Sextuple & $12(0.34 \%)$ & $0(0.00 \%)$ & $(0.00 \%)$ & $12(0.34 \%)$ \\
Septuple & $2(0.06 \%)$ & $0(0.00 \%)$ & $(0.00 \%)$ & $2(0.06 \%)$ \\
Octuple & $2(0.06 \%)$ & $0(0.00 \%)$ & $(0.00 \%)$ & $2(0.06 \%)$ \\
Total & $3566(100 \%)$ & $618(17.33 \%)$ & $2461(69.01 \%)$ & $487(13.66 \%)$ \\
\hline
\end{tabular}

\section{Age distribution of HPV infection}

Research participants were divided into 5 groups, and HPV infection rates showed an approximate U-shaped curve in terms of age. Two peaks were formed: the first HPV infection peak occurred before age 25; the second peak occurred in women older than age 55 (Figure 2 ); see Table 3 for the rate of HPV infection. The group that was younger than 25 years of age showed a higher HPV infection rate than the other age groups $\left(\chi^{2}=22.747, \mathrm{P}=0.000<0.05\right)$, There was no significant difference in the other age groups $\left(\chi^{2}=6.759, \mathrm{P}=0.08>0.05\right)$. 


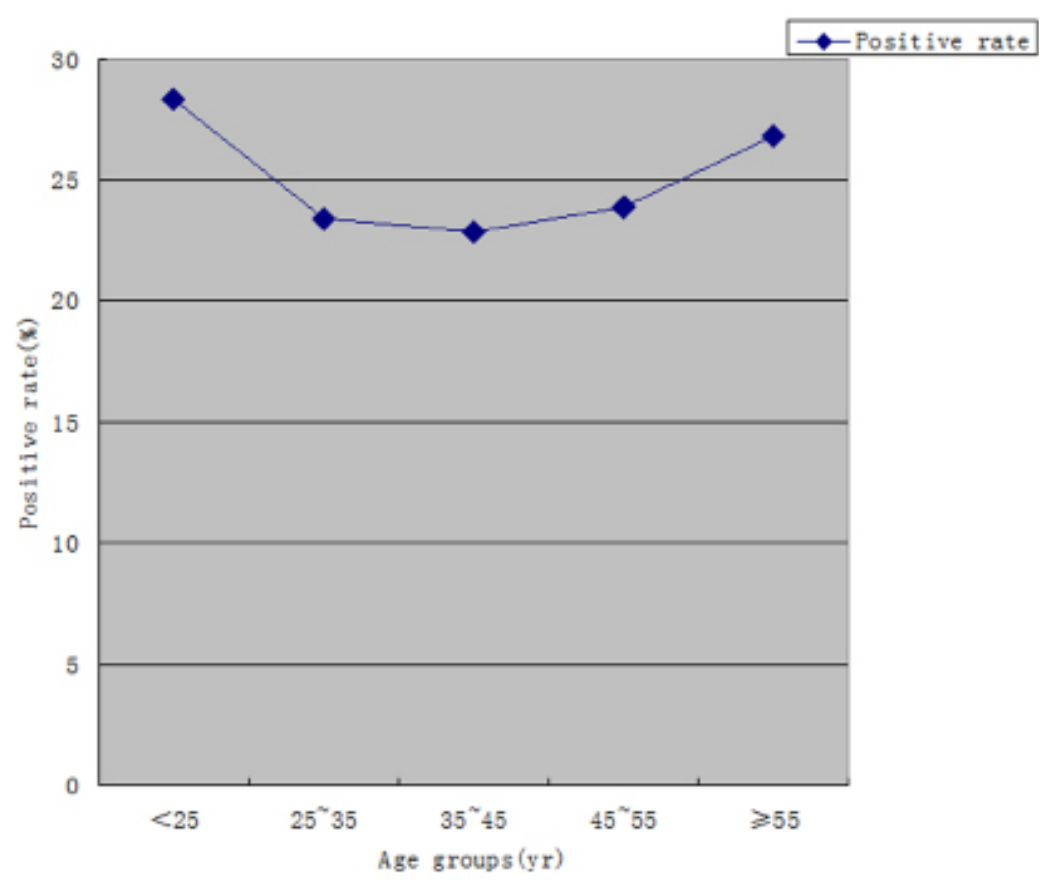

Figure 2. U-shaped relationship between age group and human papilloma virus infection rate.

Table 3. Age distribution of HPV infection.
\begin{tabular}{lcccc} 
& & & \\
\hline Age groups (years) & Cases (N) & Negative cases (N) & Positive cases (N) & Positive rate (\%) \\
\hline$<25$ & 1413 & 1013 & 400 & 28.31 \\
$25-35$ & 5318 & 4073 & 1245 & 23.41 \\
$35-45$ & 4957 & 3823 & 1134 & 22.88 \\
$45-55$ & 2298 & 1749 & 549 & 23.89 \\
$\geq 55$ & 887 & 649 & 238 & $26.83^{\mathrm{a}}$ \\
Total & 14873 & 11307 & 3566 & 23.98 \\
\hline
\end{tabular}

Compared 5 groups of infection rates: $\chi^{2}=22.747, \mathrm{P}=0.00<0.05$. Compared 4 groups of infection rates of women older than $25: \chi^{2}=6.759, \mathrm{P}=0.080>0.05$. Compared the group of women younger than 25 and women older than $55, \chi^{2}=0.593,{ }^{\text {aP }}=0.441>0.05$.

\section{DISCUSSION}

Cervical cancer is the second most common cancer and seriously threatens women's health. Currently, it also is the only cancer that has a definite cause that can be treated early and prevented - and then eradicated. High-risk HPV persistent infections are the main causes of cervical cancer and precancerous lesions (Pierce Campbell et al., 2012; Ward et al., 2012). Another study reported positive HPV infections in up to $99.7 \%$ of cervical cancer tissue specimens (Miura et al., 2006). In 2008, about 529,800 new cases of cervical cancer appeared, 275,000 women died from the disease worldwide, and it was predicted that the figure would likely rise to 410,000 cases in 2013 (Motoyama et al., 2004).

Currently, HPV has more than 200 identified genotypes, in which at least 42 are connected with reproductive tract infections (Muñoz et al., 2003). Epidemiological data showed 
that the pathogenicity of different HPV types had significant differences, such as some types of HPV being closely related to cervical cancer because persistent infection can lead to lesion progression and ultimately to cervical cancer (Motoyama et al., 2004). Therefore, some scholars believe that these potentially carcinogenic HPV genotypes can be important detection indices in the early stages of infection (Motoyama et al., 2004) and can provide the basis for early diagnosis, adequate clinical solutions, and further treatment protocols. In February 2005, the International Agency for Research on Cancer presented the differences in carcinogenicity from various types of HPV that should be considered when detecting cervical cancer resulting from HPV (Cogliano et al., 2005). In addition, clear regional distribution genotypes of HPV are significant for research and development of an HPV vaccine and prevention of cervical cancer.

Current screening for cervical cancer is carried out through cytological detection and HPV-DNA detection; both of these methods are means of secondary prevention. These methods screen out patients with early cervical lesions or cervical cancer patients at high risk as a means of early diagnosis and early cure. The most effective and safe measures should be primary prevention and a prophylactic HPV vaccine. The vaccine currently being used in clinical practice in the West has an efficient immune activity and can produce higher antibody titers to reduce persistent HPV infection and related clinical disease, thereby reducing cervical cancer. However, China is still in the developmental stages of such a vaccine and has not yet entered HPV clinical trials. China's broad geographical expanse, large population, and whether genotypes of regional HPV infection are different means there is still a need to improve epidemiological data. Developed countries generally use a bivalent (16/18) or tetravalent (16/18/6/11) HPV vaccine (Yoshikawa, 2009), with HPV 16/18 being the most common. However, according to existing epidemiological data, these vaccines do not cover the main HPV genotypes in specific areas of China. Therefore, understanding the trends of HPV occurrence in different regions and populations and developing the HPV vaccine that is appropriate for women in China will be an important step toward preventing cervical cancer in the future.

A large amount of epidemiological data shows that infection status and distribution of HPV are significantly different in different countries or regions. Clifford et al. (2005) found that the most common infection type of HPV around the world is HPV 16, followed by HPV $42,58,31,18,56,81,35,33$, and 45. Ranking second and third in Asia were HPV 33 and HPV 56, and ranking second in South America was HPV 58, whereas in Europe it was HPV 31. Rui et al. (2012) reported that the main types of infection were HPV 16, 18, 58, 56, and 52 in East Asia; HPV 52, 16, 58, 18, and 66 in Southeast Asia; and HPV 16, 18, 45, 33, and 35 in South Asia (Bhatla et al., 2008). The main types of infection in France were HPV 16, 31, 33, 45, 52, and 58 (Monsonego et al., 2012); in Korea, HPV 16, 52, 58, 18, and 56 (Lee et al., 2012); and in Taiwan, HPV 52, 16, 56, and 18 (Chen et al., 2011).

The present study showed that the main types of high-risk HPV infection in the Henan Province were HPV 16, 58, 52, 56, and 66, whereas HPV 18 showed a low infection rate. The lowest risk type was HPV 43, followed by 6,42 , and 11 . HPV 44 was not found in the Province. The most common low-risk infection was HPV 16, followed by 43, 58, 52, 56, and 66 . The low-risk HPV 43 had a very high infection rate in the Henan Province, ranking second only to 16 . The results of our study were different from reported results in other areas of the country. The main types of infection in Tibet have been reported to be HPV 16, 33, 58, 52, and 31 (Jin et al., 2009). The top 5 HPV types were reported in the following Provinces: Liaoning, HPV 16, 18, 58, 33, and 31 (Liu et al., 2010); Shandong, HPV 16, 52, 58, 18, and 11 (Liu et al., 2007); Guangxi, HPV 52, 16, 58, 53, and CP8304 (Li et al., 2012); and Zhejiang, HPV 
$52,16,58,68$, and 81 (Ye et al., 2010). However, the top 6 types in Hunan were HPV 16, 52, $58,18,6$, and 39 (Li et al., 2013). These studies show that the distribution of HPV in various regions has regional differences, although HPV 16 plays a dominant role in all regions, but the importance of the other subtypes in different regions vary. To be effective in preventing HPV infection, investigating the prevalent HPV types in various regions and developing an HPV vaccine that works for these regions is necessary.

The present study also investigated 14,873 Henan women; we found 3566 cases of HPV infection, with infection rates as high as $23.98 \%$. High-risk infection accounted for $69.01 \%$ of cases and low-risk infection accounted for $17.33 \%$; low-risk mixed with high-risk infections accounted for $13.66 \%$ of cases. HPV infection in Henan was significantly higher than in Zhejiang (13.3\%) (Ye et al., 2010) and Taiwan (16.2\%) (Chen et al., 2011), but is similar to that of Changchun (Liu et al., 2013), Hunan (Li et al., 2013), and Chaozhou in Guangdong (Chen et al., 2012), where the infection rates were 20.0, 22.6, and $24.5 \%$, respectively, but below that of Guangxi (38.9\%) (Li et al., 2012).

Mixed HPV infection is more common in molecular epidemiology of cervical cancer because certain HPV genotypes interact or have synergistic interactions that promote the development of cervical lesions (Trottier et al., 2006). In particular, the risk that occurs in high-grade lesions and invasive cancers in multiple HPV infections is greater than in a single infection (van der Graaf et al., 2002). Compared with a single HPV infection, multiple infections increase the risk of precancerous lesions (van der Graaf et al., 2002). Herrero et al. (2005) found that when HPV 16 is mixed with other types of infection, the risk of cervical lesions could greatly increase. Lee et al. (2003) further studied the relationship between multiple infections and cervical cancer and found that although a single infection increased the risk of suffering from cervical cancer by 19.9 times, multiple infections increased the risk by 31.8 times. Therefore, women with multiple infections should increase scrutiny to prevent the development of cervical cancer. The results showed that a single infection (2538 cases) occurred most often in the 3566 cases, accounting for $71.17 \%$; 1028 cases of multiple infections accounted for $28.83 \%$, of which double infection accounted for $20.11 \%$. Eight HPV subtypes were the most simultaneously detected in a multiple infection. Although the Henan Province has more single infections, multiple infections account for about a third of all infected women.

General studies suggest that high HPV infection rates in young women are related to being sexually active. As mentioned previously, the present study found that HPV infection rate showed an approximate U-shaped curve in terms of age: the infection rate of women younger than 25 years was significantly higher than in other age groups $\left(\chi^{2}=22.747, \mathrm{P}=\right.$ $0.000<0.05)$ and the infection rate in women older than 55 years also showed an increase. These results are similar to the outcomes of Yip et al. (2010) and Lee et al. (2012). As a means of analyzing the reasons for these outcomes, it may be that young women have a more active sex life; HPV infection is closely related to sexual activities. This study showed that the first peak of HPV infection occurred before age 25 years; the HPV infection rate of women older than 55 years may be associated with low immunity. The present results suggested that early sex education for young people in the Henan region should be increased, and cervical cancer screening of older women also should receive increased attention.

This study analyzed genotype distribution characteristics of HPV infection in women of the Henan Province; it was determined that HPV 16, 43, 58, 52, and 56 are the most common types of infection in the Province. If an HPV vaccine can contain these types, it will be effective in preventing HPV infection and reducing the occurrence of cervical intraepithelial 
neoplasias or cervical cancer in women in the Henan Province. At present, the relevant genotype distribution of HPV in the Province has not been studied in a large sample size, but we believe this study can provide an epidemiological basis for the development of an HPV vaccine in the Henan Province.

\section{REFERENCES}

Bhatla N, Lal N, Bao YP, Ng T, et al. (2008). A meta-analysis of human papillomavirus type-distribution in women from South Asia: implications for vaccination. Vaccine 26: 2811-2817.

Chen HC, You SL, Hsieh CY, Schiffman M, et al. (2011). Prevalence of genotype-specific human papillomavirus infection and cervical neoplasia in Taiwan: a community-based survey of 10,602 women. Int. J. Cancer 128: 1192-1203.

Chen Q, Luo ZY, Lin M, Lin QL, et al. (2012). Prevalence and genotype distribution of human papillomavirus infections in women attending hospitals in Chaozhou of Guangdong province. Asian Pac. J. Cancer Prev. 13: 1519-1524.

Clifford GM, Gallus S, Herrero R, Muñoz N, et al. (2005). Worldwide distribution of human papillomavirus types in cytologically normal women in the International Agency for Research on Cancer HPV prevalence surveys: a pooled analysis. Lancet 366: 991-998.

Cogliano V, Baan R, Straif K, Grosse Y, et al. (2005). Carcinogenicity of human papillomaviruses. Lancet Oncol. 6: 204.

Herrero R, Castle PE, Schiffman M, Bratti MC, et al. (2005). Epidemiologic profile of type-specific human papillomavirus infection and cervical neoplasia in Guanacaste, Costa Rica. J. Infect. Dis. 191: 1796-1807.

Jin Q, Shen K, Li H, Zhou XR, et al. (2009). Prevalence of human papillomavirus infection in women in Tibet Autonomous Region of China. Zhonghua Fu Chan Ke Za Zhi 44: 898-902.

Lee EH, Um TH, Chi HS, Hong YJ, et al. (2012). Prevalence and distribution of human papillomavirus infection in Korean women as determined by restriction fragment mass polymorphism assay. J. Korean Med. Sci. 27: 1091-1097.

Lee SA, Kang D, Seo SS, Jeong JK, et al. (2003). Multiple HPV infection in cervical cancer screened by HPVDNAChip. Cancer Lett. 198: 187-192.

Li H, Zhang J, Chen Z, Zhou B, et al. (2013). Prevalence of human papillomavirus genotypes among women in Hunan province, China. Eur. J. Obstet. Gynecol. Reprod. Biol. 170: 202-205.

Li HM, Liang GJ, Yin YP, Wang QQ, et al. (2012). Prevalence and genotype distribution of human papillomavirus infection among female sex workers in Guangxi, China: implications for interventions. J. Med. Virol. 84: 798-803.

Liu M, Wang CX, Deng XM, Wang LS, et al. (2007). Study on the genotyping of human papillomavirus using a new DNA liquid chip in women of high-risk group of Shandong province. Zhonghua Liu Xing Bing Xue Za Zhi 28: 487-490.

Liu W, Wu EQ, Yu XH, Feng LH, et al. (2013). Detection of human papillomavirus genotypes associated with mucopurulent cervicitis and cervical cancer in Changchun, China. Int. J. Gynaecol. Obstet. 12: 124-126.

Liu X, Zhang S, Ruan Q, Ji Y, et al. (2010). Prevalence and type distribution of human papillomavirus in women with cervical lesions in Liaoning Province, China. Int. J. Gynecol. Cancer 20: 147-153.

Miura S, Matsumoto K, Oki A, Satoh T, et al. (2006). Do we need a different strategy for HPV screening and vaccination in East Asia? Int. J. Cancer 119: 2713-2715.

Monsonego J, Zerat L, Syrjanen K, Zerat JC, et al. (2012). Prevalence of type-specific human papillomavirus infection among women in France: implications for screening, vaccination, and a future generation of multivalent HPV vaccines. Vaccine 30: 5215-5221.

Motoyama S, Ladines-Llave CA, Luis Villanueva S and Maruo T (2004). The role of human papilloma virus in the molecular biology of cervical carcinogenesis. Kobe J. Med. Sci. 50: 9-19.

Muñoz N, Bosch FX, de Sanjosé S, Herrero R, et al. (2003). Epidemiologic classification of human papillomavirus types associated with cervical cancer. N. Engl. J. Med. 348: 518-527.

Pierce Campbell CM, Menezes LJ, Paskett ED and Giuliano AR (2012). Prevention of invasive cervical cancer in the United States: past, present, and future. Cancer Epidemiol. Biomarkers Prev. 21: 1402-1408.

Trottier H, Mahmud S, Costa MC, Sobrinho JP, et al. (2006). Human papillomavirus infections with multiple types and risk of cervical neoplasia. Cancer Epidemiol. Biomarkers Prev. 15: 1274-1280.

van der Graaf Y, Molijn A, Doornewaard H, Quint W, et al. (2002). Human papillomavirus and the long-term risk of cervical neoplasia. Am. J. Epidemiol. 156: 158-164.

Ward KK, Shah NR, Saenz CC, McHale MT, et al. (2012). Changing demographics of cervical cancer in the United States (1973-2008). Gynecol. Oncol. 126: 330-333.

Ye J, Cheng X, Chen X, Ye F, et al. (2010). Prevalence and risk profile of cervical Human papillomavirus infection in Zhejiang Province, southeast China: a population-based study. Virol. J. 7: 66. 
Yip YC, Ngai KL, Vong HT, Tzang LC, et al. (2010). Prevalence and genotype distribution of cervical human papillomavirus infection in Macao. J. Med. Virol. 82: 1724-1729.

Yoshikawa H (2009). Progress and challenges on HPV vaccination. Uirusu 2: 243-248. 